\title{
Meta-aprendizagem e Ciência da Informação: uma reflexão sobre o ato de aprender a aprender
}

\section{Dulce Amélia de Brito Neves}

\begin{abstract}
Doutora em Ciência da Informação; Professora Adjunta do Departamento de Biblioteconomia e Documentação da UFPB.
\end{abstract}

Apresenta uma reflexão sobre a meta-aprendizagem. Analisa aportes teóricos sobre o uso da metacognição na aprendizagem. Evidencia a importância do ensino da metacognição visando ao desenvolvimento pelo aluno de métodos eficazes para lidar com a informação proveniente do meio e com seus próprios processos mentais. Enfatiza o aprendizado voltado para atividades metacognitivas que têm produzido resultados satisfatórios no desempenho escolar. Apresenta uma abordagem da Ciência da Informação destinada àqueles que fazem parte dos Sistemas de Recuperação.

Palavras-chave: Meta-aprendizagem; Estratégias metacognitivas; Mapas cognitivos; Mapas conceituais; Ciência da Informação.

\section{Meta-learning: A reflection on the act of learning to learn}

The article presents a reflection on meta-learning, analyzes theoretical support about the use of metacognition in learning, stresses the importance of teaching metacognition for learner to develop some efficient methods to deal with the information obtained from the environment and the mental processes involved. It emphasizes the learning in metacognitive activities that have produced satisfactory performance in schools. An Information Science approach directed to those at the Information Systems is also presented.

Keywords: Metacognition; Metacognitive strategies; Learning; Conceptual maps; Cognitive maps, Information Science. 


\section{Introdução}

Nas diversas áreas do conhecimento está ocorrendo, desde meados do século $X X$, um movimento em direção a teorias, relativamente recentes, que focalizam a emergência de pesquisas sobre o desempenho mental dos indivíduos. Pretende-se resposta para a inquietante questão: como a nossa mente funciona? Não se busca saber apenas o funcionamento fisiológico do cérebro, mas os meandros da origem do comportamento e atitudes dos seres humanos.

Observamos que os indivíduos estão em permanente estado de aprendizagem, quer estejam sós ou em sociedade. A reflexão sobre os acontecimentos, consciente ou inconscientemente, nos leva a uma interação entre o conhecimento adquirido e o meio ambiente, tendo em vista a nossa sobrevivência. A nossa mente encontra-se em atividade, estejamos acordados ou dormindo. Durante nosso repouso ocorre a organização de todas as informações adquiridas durante o dia para uso futuro.

Existem momentos em que agimos aparentemente de modo automático, em outros estimulamos a emergência do conhecimento adquirido e armazenado em nossa memória, na busca de soluções para os problemas que se apresentam em nosso cotidiano. Nesse sentido, estamos usando uma auto-regulação na recuperação das informações necessárias para aquela situação especifica. Apesar de usarmos este processo conscientemente, muitas vezes não nos damos conta do potencial presente na gestão cognitiva e no controle que podemos exercer sobre os nossos processos mentais.

Tomarmos consciência de como podemos gerenciar nossas atividades, a partir de estratégias que não apenas refletem como realizar as tarefas imediatas, mas também como fazer para adquirirmos novas informações, é "aprender a aprender" (RIBEIRO, 2003; ALVAREZ, 2004, entre outros autores).

\section{Meta-aprendizagem}

A cognição é compreendida como os processos mentais inconscientes de uma pessoa, enquanto que a metacognição é a gestão dos processos cognitivos pelo indivíduo, referida por Flavell (1979) como o "pensamento sobre o pensamento", ou seja, o controle consciente sobre um fenômeno cognitivo. Flavell (1979), Pressley e Afflerbach (1995), por exemplo, demonstraram que as pessoas usam de modo progressivo as habilidades metacognitivas. Tais habilidades envolvem a compreensão e o monitoramento consciente dos processos cognitivos, por meio da modificação de algumas atividades cognitivas, adequando-as durante o desempenho de tarefas. A aprendizagem é uma ação metacognitiva, já 
que é um processo consciente de aquisição de informação, transformando-a em conhecimento.

A metacognição pressupõe o uso da monitoração ou auto-regulação. A monitoração pode ser explicada como o acompanhamento e avaliação de um determinado itinerário, nem sempre seqüencial, seguido pelo indivíduo, em busca da solução para um problema. A monitoração também se denomina regulação, por proporcionar um equilíbrio, o mais harmônico possível, entre as várias estratégias que estão sendo adotadas em um determinado momento, juntamente com todos os componentes necessários para o sucesso na realização de uma tarefa.

As estratégias metacognitivas são utilizadas por meio da monitoração, ou seja, estamos sempre avaliando o estado da nossa compreensão do item que estamos lendo, buscando alcançar o seu completo entendimento, do nosso ponto de vista, levando também em consideração o ponto de vista do autor. Por isso, a leitura é considerada como algo de difícil implementação. A monitoração não é totalmente linear, pois durante a solução de um problema, a todo momento procuramos garantir que o caminho percorrido até então seja seguro e nos aproxime da nossa meta. Caso percebamos que as estratégias adotadas não nos levem à solução desejada, retornamos àquele ponto questionado e buscamos adotar uma estratégia mais adequada. Estratégias metacognitivas são solicitadas para estimular os progressos cognitivos, monitorando-os e regulando-os.

É atribuído a Flavell (1979) o uso do termo metacognição pela primeira vez, no sentido do conhecimento sobre um fenômeno cognitivo que ocorre por meio da interação de "conhecimento metacognitivo" e "experiências metacognitivas". O conhecimento metacognitivo representa o nosso conhecimento de mundo que diz respeito à consciência que temos do que sabemos e do que achamos que as pessoas sabem.

As experiências metacognitivas, segundo Flavell (1979), ocorrem em situações de pensamento altamente consciente como, por exemplo, em novas situações nas quais os passos são da maior importância, pois exigem planejamento antecipado e avaliação posterior; ou quando as decisões e ações são cruciais, envolvendo componentes de risco. Atravessar uma rua ou mesmo fazer uma prova são exemplos desse tipo de situação. O grau de risco é determinado pela pessoa envolvida.

Tanto o conhecimento quanto a experiência metacognitiva envolvem três variáveis: pessoa, tarefa e estratégia. A variável pessoa refere-se ao conhecimento geral de um indivíduo sobre a sua própria cognição e a de outras pessoas. A variável tarefa diz respeito ao conhecimento das pessoas sobre a natureza das tarefas, o tipo de processamento que deve ser adotado, e o gerenciamento necessário para o sucesso no desempenho de um determinado empreendimento. A variável estratégia abrange o conhecimento sobre as estratégias prováveis de serem ativadas para a obtenção de determinados sub-objetivos e objetivos nos diferentes tipos de empreendimentos.

Alvarez (2004) evidencia a importância da aprendizagem através do uso de estratégias de resolução de problemas com o emprego de 
estratégias armazenadas na memória, como uma forma mais eficaz de selecionar métodos de trabalho mais apropriados. Em várias situações de aprendizagem, o uso e o treinamento em estratégias metacognitivas levará os indivíduos a implementarem suas tarefas de modo mais eficiente (PRESSLEY, 2000). As estratégias metacognitivas são utilizadas para o conhecimento e controle de atividades a serem desenvolvidas. Estas estratégias potencializam a consciência de si próprio enquanto processador da informação (por exemplo, saber se aprende melhor lendo em voz alta) e permite a escolha da estratégia melhor adequada à situação (fazer apontamentos ou criar esquemas gerais sobre a informação).

A meta-aprendizagem, como atualmente é trabalhada, desenvolve o conhecimento de estratégias que melhoram ou aperfeiçoam a aprendizagem dos conteúdos, e ajudam a selecionar e adequar estas estratégias a cada situação. Nesse sentido, tendo em vista que esse conhecimento vai além da abordagem metacognitiva, gostaríamos de propor o seguinte conceito: meta-aprendizagem é a gestão de conteúdos cognitivos e metacognitivos que possam implementar e enriquecer $o$ conhecimento dos indivíduos direcionados a um determinado objetivo. Em nossa opinião, a metacognição é apenas um dos aspectos de gestão cognitiva utilizados na meta-aprendizagem, pois existem também os mapas cognitivos e os mapas conceituais. O ensino e treinamento para a realização desses mapas podem dar uma grande contribuição para o incremento da aprendizagem, principalmente no que se refere às TICs.

O pensamento humano é construído por redes e associações. O modo de pensar dos seres humanos não é linear, pois produz novos saberes de modo semelhante a uma rede hipertextual. Um novo saber se conecta com um saber já existente podendo ser atualizado, refutado e/ou reelaborado. Tudo depende da nossa produção de sentidos, de como constituímos os significados. O conhecimento pode ser armazenado de duas formas: lingüística (semântica), que inclui fala e leitura; e nãolingüística, que inclui imagens mentais e até mesmo sentidos físicos como olfato, audição, tato, etc. As representações não-lingüísticas podem complementar as representações lingüísticas no processo de aprendizagem. Nas representações não-lingüísticas inclui-se o mapeamento de conceitos. Na abordagem da teoria construtivista o sujeito constrói seu conhecimento e significado a partir de relações entre diversos elementos, os quais viabilizam a sistematização de conceitos novos em novos significados, ou reformulam um já existente.

Nesse sentido, Ausubel (1982) ressalta o conteúdo verbal da aprendizagem, enfatizando a aquisição e retenção de conhecimentos de modo "significativo", que fundamentou a sua teoria denominada "aprendizagem significativa". Para esse autor, a aprendizagem não depende necessariamente da motivação. Ou seja, quando se aprende algo há uma satisfação inicial que faz emergir um desejo de continuidade daquela situação. Assim, Ausubel, Novak e Hanesian (1980) propuseram que esquemas e diagramas são úteis para ilustrar a teoria da aprendizagem significativa. 
$\mathrm{Na}$ década de 60 surgiram algumas técnicas de mapeamento de informação. Novak (1988) criou os mapas conceituais que consistem em um esquema para mapear, de forma hierárquica, os conceitos presentes em um conhecimento, e cujo objetivo é representar relações significativas entre conceitos na forma de proposições ${ }^{1}$. Essas proposições unem conceitos através de palavras que os relacionam, constituindo uma unidade semântica. Os mapas seguem uma estrutura hierárquica e têm como referência o grau de generalidade e o grau de inclusão de um conceito. Além disso, podem auxiliar no planejamento de currículos e no planejamento de diversas atividades do processo ensino-aprendizagem, inclusive na construção de hipertextos educacionais.

Os mapas cognitivos ${ }^{2}$ são usados na modelagem computacional para delinear e representar o pensamento humano. O termo mapa cognitivo refere-se a uma representação do ambiente no cérebro. É uma atividade que os cientistas relacionam como uma das principais funções do cérebro. Os mapas envolvem conceitos e relações entre conceitos que são utilizados pelos indivíduos para compreenderem o seu ambiente e dar-lhe sentido. Para Swan (1997), os mapas cognitivos são representações, esquemas ou modelos mentais construídos pelos indivíduos em suas interações com o meio ambiente social ou de aprendizagem.

O mapeamento cognitivo é um conjunto de técnicas de pesquisa voltado para a identificação dos elementos que integram esses mapas ou modelos construídos pelos indivíduos e partilhados, em maior ou menor grau, por outros indivíduos. Essas técnicas envolvem formas de retratar graficamente as crenças subjetivas acessadas, permitindo a visualização das relações significativas identificadas (COSSETTE; AUDET 1994). Nesse sentido, um mapa cognitivo expressa a representação mental do discurso enunciado pelo sujeito, do qual o pesquisador elabora uma representação gráfica.

A automatização de tarefas permite o processamento de um volume muito mais significativo de dados e de tarefas repetitivas, mas não se deve esquecer que a natureza humana tem os seus próprios limites face a um processamento automatizado de dados, que se revela quantitativa e qualitativamente inadequado à capacidade humana. Nesse sentido, para uma melhor interação entre o ser humano e as tecnologias digitais existe a ergonomia cognitiva. É uma disciplina que estuda os processos cognitivos em situação de trabalho, contribuindo para o estabelecimento de novos ambientes de trabalho, nos quais ocorreu uma crescente automatização das diferentes tarefas.

O emprego da meta-aprendizagem na educação desde a mais tenra idade proporcionará maior segurança ao educador e, até mesmo, agilidade no aprendizado por parte dos alunos. Mesmo para os adultos, o

As proposições são representações conceituais expressadas verbalmente em linguagem natural, ou seja, idéias que podem ser associadas a certas características, estabelecendo conexões com outras idéias. (NEVES, 2004).

2 Na psicologia cognitiva temos a definição de mapas cognitivos como: "representações mentais do ambiente físico, particularmente quanto às relações espaciais entre os objetos no ambiente" (STERNBERG, 2000, p. 434). 
uso da metacognição, dos mapas conceituais e dos mapas cognitivos trará novas possibilidades para o estabelecimento de novas rotas em direção ao objetivo almejado. Já a abordagem da ergonomia cognitiva poderá contribuir para que o educador conheça melhor os educandos, adaptando os métodos de ensino ao perfil dos indivíduos com quem lidará futuramente.

\section{Meta-aprendizagem e a Ciência da Informação}

\section{A Ciência da Informação tem como principal função}

produzir conhecimentos que contribuam para a solução de problemas relacionados à organização de sistemas de informação especializados na incorporação, sistematização, disseminação e recuperação da informação (NEVES; MOURA, 2002, p.177).

Freire (2006, p. 16) evidencia que "um dos objetivos da ciência da informação seria contribuir para a informação se tornar, cada vez mais, um elemento de inclusão social, trazendo desenvolvimento para as pessoas e nações".

Além destes pontos levantados, percebemos que cabe à $\mathrm{CI}$, através de novas pesquisas, buscar "descobrir novas formas de pensar e agir de maneira ativa nos diversos processos de comunicação na sociedade" (FREIRE, 2006, p. 17). Dentre as diversas abordagens trabalhadas pelos pesquisadores de CI, o fenômeno da informação e sua relação com os processos cognitivos dos usuários de sistemas conceituais começam a se ampliar (BIOLCHINI, 2001).

Nesse sentido, De Mey (1982, p. 4) referindo-se ao ponto de vista cognitivo na CI afirma que "cada ato de processamento da informação, seja ele perceptivo ou simbólico, é mediado por um sistema de categorias e conceitos os quais, para o mecanismo de processamento da informação, constituem um modelo de mundo".

Ao refletirmos sobre esta afirmativa vemos que, enquanto o uso da percepção possibilita a emergência de conhecimento anterior, os sentidos e os signos dão referências ligadas às representações inerentes a cada individuo. O processamento de certa informação passa por uma organização e pela produção de elementos a partir de uma seqüência de instruções que já temos armazenada. Assim, a informação é trabalhada cognitivamente, ou seja, efetua-se a manipulação dos dados, atendendo a um tipo de processamento executivo, cujas instruções abrangem a entrada, a verificação, o armazenamento, a recuperação, a transformação e a produção de novas informações, a partir da incorporação dos dados iniciais. Por exemplo: a visão do fogo ou a sensação de calor incomum nos leva imediatamente a uma atitude de defesa instintiva; provavelmente correremos para um lugar mais seguro (reação cognitiva). Isto se deve ao fato de termos aprendido que o fogo não apenas queima, mas também 
mata. No entanto, quando refletimos metacognitivamente sobre a situação, tomamos as providências adequadas, usamos um extintor e/ou chamamos o corpo de bombeiros (reação metacognitiva).

Após a organização cognitiva de uma nova informação, passa-se ao "tratamento" metacognitivo desses dados, que pressupõe um planejamento mental usando estratégias e abordagens conscientes formuladas na mente. Nem todos os elementos resultantes são aproveitados, alguns deles podem ser armazenados para uso posterior ou simplesmente descartados. Outros componentes gerados pela nova informação possibilitam a transformação daquela informação desconhecida em um novo conhecimento. Podemos exemplificar com uma corrente de fluxo emergente.

Em síntese, o uso da cognição em conjunto com a metacognição possibilita que elementos de informação ou representação de um determinado fato sejam armazenados, processados quase automaticamente. Estes elementos se reúnem e são tomados como base para a formação de novas idéias, possibilitando a transformação da informação original (bruta) em conhecimento incorporado ao conjunto de bens armazenados em nossa mente.

Flavell (1979, p. 906) define metacognição como "o conhecimento sobre um fenômeno cognitivo" que ocorre por meio da interação entre "conhecimento metacognitivo" e "experiências metacognitivas". O conhecimento metacognitivo representa o nosso conhecimento de mundo, que diz respeito à consciência que temos do que sabemos e do que achamos que as pessoas sabem. Já as experiências metacognitivas ocorrem em situações de pensamento altamente consciente como, por exemplo, em novas situações, em que cada passo é da maior importância, exige planejamento antecipado e avaliação posterior; ou em situações em que as decisões e ações são cruciais, envolvendo componentes de risco, seja a realização de uma prova, uma cirurgia neurológica ou a travessia de uma rua movimentada. O componente risco está intimamente ligado à avaliação subjetiva do indivíduo.

Existem duas variáveis relacionadas ao uso do conhecimento e da experiência metacognitiva: metas e estratégias. As metas dizem respeito ao conhecimento que as pessoas têm sobre a natureza das tarefas, o tipo de processamento que deve ser adotado e o gerenciamento necessário para o sucesso no desempenho da tarefa. Já a variável estratégia abrange o conhecimento sobre as estratégias prováveis de serem efetuadas para a obtenção de determinados resultados nos diferentes tipos de empreendimentos cognitivos.

Como a metacognição é o conhecimento sobre o conhecimento, ela é um ato passível de ser gerenciado pelos indivíduos que, ao ficarem cientes destas habilidades, sofrem modificação cognitiva nas abordagens das tarefas. Ou seja, as demandas de certa tarefa mudam, são determinadas agora pelos próprios indivíduos criando novas atitudes em seu cotidiano, até mesmo no enfrentamento de problemas de ordem pessoal. 
Outros estudos buscam um entendimento do funcionamento da nossa mente, como o dos mapas, que são vistos como descrições da imagem mental de uma pessoa a respeito de um objeto ou situação e envolvem conceitos e relações entre conceitos. Os mapas não são representações estáticas do ambiente, são sempre atualizados a partir das experiências do sujeito; vão sendo reconstruídos pelo processo de aprendizagem. Como já existe uma interação entre os indivíduos e os ambientes virtuais, a ergonomia cognitiva se propõe estudar os processos cognitivos dos indivíduos em interação com o ambiente, apontando novas possibilidades de interação entre o homem e a máquina, dirimindo conflitos.

Os mapas conceituais se referem, por exemplo, à representação, e indicam relações entre conceitos na forma de proposições. Seu uso na CI é de grande interesse, pois possibilita a construção da representação conceitual dos processos mentais dos indivíduos em suas buscas e/ou em seu trabalho cotidiano em sistemas de informação, visto que o mapeamento de um conhecimento permite conhecer a hierarquia do conceito e sua formação na mente. Já os mapas cognitivos pretendem traçar a representação do ambiente no cérebro, e envolve representações, modelos mentais ou esquemas que os indivíduos constroem a partir de suas interações no ambiente social e durante a aprendizagem (SWAN, 1997).

Há inúmeras habilidades cognitivas a serem compreendidas e desenvolvidas nos seres humanos em interação, no âmbito dos sistemas de informação. Ao aliarmos pesquisas relativas à metacognição, aos mapas conceituais, aos mapas cognitivos e à ergonomia cognitiva em sistemas de informação, poderemos focalizar o profissional da informação como, também, as interações entre o sistema e seus usuários, pois as atividades cognitivas estão presentes nas práticas cognitivas de busca e recuperação da informação.

Nesse sentido, descortinam-se várias possibilidades para os profissionais e usuários de unidades de informação, que poderão trazer o conhecimento de habilidades, tanto no tratamento da informação quanto no cotidiano do bibliotecário de referência. Além disso, como o processo de indexação é um processo intelectual, que está intimamente ligado à abordagem do processamento da informação na psicologia cognitiva, a metacognição pode instruir os indexadores a como ler estrategicamente, além de instruí-los a fim de que desenvolvam habilidades que facilitarão o seu desempenho.

Os mapas conceituais podem ser aplicados a estudos relativos à representação mental de proposições e conceitos dos indivíduos em suas buscas e/ou em seu trabalho cotidiano em sistemas de informação, visto que o mapeamento de um conhecimento permite conhecer a hierarquia do conceito e sua formação na mente. Os mapas cognitivos pretendem traçar a representação do ambiente no cérebro envolvendo representações, modelos mentais ou esquemas que os indivíduos constroem a partir de suas interações no ambiente social e durante a aprendizagem. 
Ao aliarmos a metacognição (gestão dos processos cognitivos) aos mapas cognitivos e conceituais, surgem inúmeras possibilidades para 0 avanço da compreensão de procedimentos e atitudes dos que fazem e usam as unidades de informação. Por exemplo: estudos já existentes relacionados ao desempenho metacognitivo do indexador durante a leitura para fins de indexação Neves, 2006; Fujita e Rubi (2006). Estas pesquisas poderiam ser alargadas com estudos relativos aos mapas conceituais, mapas cognitivos e ergonomia cognitiva.

O mapa cognitivo do processo de indexação, por exemplo, poderia explicitar certos procedimentos como a análise de assunto, a leitura e a elaboração do conceito, que são as três principais fases da indexação. Além disso, poderia indicar o caminho percorrido pelo profissional da informação, ajudando-o a organizar e estruturar melhor seu trabalho. A ação de construir (e debater) os mapas cognitivos pode ajudar grupos a converter conhecimento tácito em conhecimento explícito. Já os mapas conceituais podem mostrar como os indexadores selecionam e elaboram o "conceito" atribuído ao documento analisado. Os mapas conceituais são ferramentas importantes para a elaboração de novas metodologias de trabalho. Poderíamos dizer que seria um repensar sobre o processo de indexação.

No que diz respeito à ergonomia cognitiva, que estuda os processos cognitivos em interação com os ambientes, uma vez aplicada às unidades de informação, poderíamos realizar pesquisas no sentido de estabelecer uma interação harmônica entre o Sistema de Recuperação da Informação (SRI) e seus usuários e profissionais.

Tais investigações poderão contribuir para tornar as unidades de informação mais apropriadas aos seus usuários, como também para permitir uma maior compreensão das necessidades dos profissionais da informação, já que ajudarão a perceber como eles sentem e reagem ao ambiente. Além disso, proporcionarão a construção de instrumentos de controle terminológicos, adequados a cada área e sub-área do conhecimento, em permanente interação com a linguagem do usuário, dos seus organizadores e indexadores que deles farão uso.

Estas adequações serão possíveis a partir do estudo da sociocognição inerente ao indexador, ou seja, do compartilhamento de uma cognição profissional inerente a estes profissionais. Com isto será possível diminuir barreiras e ruídos na recuperação da informação, facilitando a assimilação da informação por parte do usuário e, consequentemente, a transformação dessa informação em conhecimento.

Segundo Hjorland e Albrechtsen, 1995 apud Freire (2006, p. 16),

$O$ estudo dessas comunidades, e de outras que não se situam no campo científico, se configura como um novo espaço para a abordagem cognitivista na ciência da informação, não mais baseado nos processos mentais do indivíduo (como em Belkin, por exemplo), mas levando em consideração o contexto social no qual o processo de informação ocorre. 


\section{Considerações Finais}

Com a presente reflexão sobre as novas possibilidades, que vão além da metacognição, pretendemos colocar em discussão trabalhos transdisciplinares (NEVES e CRUZ, 2000) que indiquem novos rumos aos estudos referentes à metacognição e à aprendizagem no cotidiano dos indivíduos.

No uso de novos métodos e técnicas na busca por uma melhor adequação das unidades de informação em interção com seus usuários e com os profissionais da informação, acreditamos que pesquisas utilizando a metacogção, os mapas conceituais, os mapas cognitivos e a ergonomia cognitiva possam levar à compreensão de como a informação se transforma em conhecimento.

Almejamos colocar em pauta a discussão sobre a metaaprendizagem. Não apenas como sendo o estudo dos processos metacognitivos, mas também sua afinidade com as pesquisas, o uso e o ensino de mapas conceituais na construção da aprendizagem e no esclarecimento de como os indivíduos constroem seu conhecimento; dos mapas cognitivos nas investigações sobre a interação dos seres humanos com os ambientes virtuais, culminando com os estudos da ergonomia cognitiva que busca uma melhor interação entre o ser humano e as novas tecnologias.

No que se refere à Ciencia da Informação, nesessitamos de estudos que viabilizem o entendimento das práticas cognitivas de busca e recuperação da informação, considerando-se também os profissionais da informação, na pessoa dos bibliotecários e arquivistas, entre outros.

Nesta perspectiva, aprender tem um sentido mais amplo. Uma completude que a própria natureza dos seres humanos, em permanente construção, proporciona, na interação com o ambiente que os cercam. Para finalizarmos esse artigo reproduziremos a seguinte reflexão:

Nossas vidas consistem de um conjunto de comportamentos e movimentos num ambiente espacial. Cada dia nós fazemos centenas de complexas escolhas e decisões espaciais. Numa vasta maioria de casos confiamos em referências externas tais como mapas para fazer escolhas que dependem previamente da compreensão espacial do mundo que vivemos. Nós precisamos de representações espaciais do ambiente em nossas mentes ... precisamos de nossos mapas cognitivos... (DIÁRIO..., 2005, p. 5).

\section{Referências}

ALVAREZ, M. J. Aprender a aprender: estratégias de aprendizagem e auto-regulação cognitiva.Disponível em: <http://www.geocities.com/mjalvarezpt/>. Acesso em: 24 out. 2004. 
AMORETTI, M. S. M.; TAROUCO, L. Mapas conceituais: modelagem colaborativa do conhecimento. Revista Informática na Educação: Teoria \& Prática, v.3 n.1, set. 2000.

AUSUBEL, D. P. A aprendizagem significativa: a teoria de David Ausubel. São Paulo: Moraes, 1982.

Aquisição e retenção de conhecimentos: uma perspectiva cognitiva. Lisboa: Editora Plátano, 2003.

AUSUBEL, D. P.; NOVAK, J.D.; HANESIAN, H.. Psicologia educacional. Rio de Janeiro: Interamericana, 1980.

BELKIN, N. J. Cognitive models and information transfer. Social Science Information Studies, n. 4, 1984.

Some soviet concepts of Information for Information Science. Journal of the American Society for Information Science, p. 56-64, 1975.

CHAN, C. K. K. Constructive activity in learning from text. American Educational Research Journal, n. 29, p. 97-98, 1992.

COSSETTE, P.; AUDET, M. Mapping of an idiosyncratic schema. Journal of Management Studies, v.29, n.3, pp. 321-347, 1992.

DE MEY, M. The cognitive paradigm: an integrated understanding of science development. Chicago: University of Chicago, 1982.

DIÁRIO de bordo. Síntese de algumas abordagens sobre aprendizagem. Disponível em: Disponível em: <http://www.clubedoprofessor.com.br/diariodebordo/Textop5a.html>. Acesso em: 19 out. 2005.

FLAVELL, J. H. Metacognition and cognitive monitoring: a new area of cognitive-developmental inquiry. American Psychologist, v. 34, p. 906$911,1979$.

FREIRE, G. H. de A. Ciência da Informação: temática, histórias e fundamentos. Perspectivas em Ciência da Informação, Belo Horizonte, v.11, n. 1, p. 6-19, jan./abr. 2006.

FUJITA, M. S. L.; RUBI, M. P. Um modelo de leitura documentária para a indexação de artigos científicos: princípios de elaboração e uso para a formação de indexadores. DataGramaZero - Revista de Ciência da Informação, v.7, n.3, jun. 2006. Disponível em: <http://www.dgz.org.br/jun06/F | art.htm>. Data de acesso: 10 maio 2007.

HJØRLAND, B.; ALBRECHTSEN, H. Toward a new horizon in information science: domain-analysis. Journal of the American Society for Information Science, v. 46, n. 6, 1995 apud FREIRE, G. H. de A. Ciência da Informação: temática, histórias e fundamentos. Perspectivas em Ciência da Informação, Belo Horizonte, v.11, n. 1, p. 6-19, jan./abr. 2006.

JOHNSON-LAIRD, P. Mental models. Cambridge, MA: Harvard University Press, 1983. 
KITSCH, W., VAN DIJK, T. A. Toward a model of text comprehension and production. Psychological Review, v. 85, n. 5, p. 363-394, Sep, 1978.

LASZLO, E. et al. The evolution of cognitive maps: new paradigms for the twenty-first century. Amsterdam: Gordon and Breach, 1995.

NEVES, D. A. de B. Leitura e metacognição: uma experiência em sala de aula. Encontros Bibli, n. 24, 2007. Disponível em: <http://www.encontrosbibli.ufsc.br/regular.html> Acesso em: 20 set. 2007.

. Ciência da informação e cognição: uma abordagem do processamento da informação. Ciência da Informação, Brasília, v. 34, n. 3, p. 39-44, 2006. Disponível em: http://www.ibict.br/cienciadainformacao/viewarticle.php?id=747\&layout=abstract

- La verbalización como registro para análisis en la investigación sobre lectura. Anales de documentación, Revista de Biblioteconomía y Documentación, v.9, 2006. Disponível em: <http://www.um.es/fccd/anales/ad09/ad0900.html >. Acesso em: Acesso em: 20 set. 2007.

Aspectos metacognitivos na leitura do indexador. 130 f. 2004. Tese (Doutorado) - Escola de Ciência da Informação, Universidade Federal de Minas Gerais - UFMG, Belo Horizonte, 2004.

NEVES, D. A. de B., MOURA, M. A. Ciência da Informação, semiótica e cognição: interseções. Athos \& Ethos, v.2, n.1, p.175 - 198, 2002.

NEVES, D. A. de B., CRUZ, E. B. Transacionando com os campos do saber. In: ENCONTRO NACIONAL DE PESQUISA EM CIÊNCIA DA INFORMAÇÃO ENANCIB, 4., 2000, Brasília. Anais... Brasília: UnB, 2000.

NOVAK, J. D. Teoría y practica de la educación. Espanha: Ed.Alianza, 1988.

NOVAK, J. D.; GOWIN, D. B. Aprender a aprender. Lisboa: Platano Edições Técnicas, 1996.

PARIS, S.; WINOGRAD, P. How metacognition can promote academic learning and instruction. In: JONES, B; IDOL, L. (Orgs.). Dimensions of thinking and cognitive instruction. Hillsdale, N. J.: Erlbaum, 1990. p. 333-349.

PRESSLEY, M. What should comprehension instruction be to instruction of? In: KAMIL, M. et. al. (Eds). Handbook of reading research. New Jersey: Lawrence Erlbaum, 2000. p. 545-561.

Beyond direct explanation: transactional instruction of reading comprehension strategies. Elementary School Journal, n. 92, p. 511-554, 1992.

PRESSLEY, M.; AFFLERBACH, P. Verbal protocols of reading: the nature of constructively reading. New Jersey: Lawrence Erlbaum, 1995.

RIBEIRO, C. Metacognição: um apoio ao processo de aprendizagem. Psicologia Reflexão e Critica, Porto Alegre, v.16, n.1, p. 109-116, 2003.

STERNBERG, R. J. Psicologia cognitiva. Porto Alegre: Artmed, 2000.

SWAN, J. Using cognitive mapping in management research: decisions about technical innovation. British Journal of Management, n. 8, p. 183198, 1997.

VAN DIJK, T. A.. Cognição, discurso e interação. São Paulo: Contexto, 1999. 
VIGOTSKI, L. S.; LURIA, A. R.; LEONTIEV, A. N.. Linguagem, desenvolvimento e aprendizagem. São Paulo: Ícone, 1998. 